\title{
Numerical Integration and Complex Trend Term Elimination of Acceleration Signal in Fault Diagnosis
}

\author{
YANG Yi-zhou ${ }^{1, a}$, JIANG Dong-xiang ${ }^{1, b}$ \\ ${ }^{1}$ Gas Turbine Institute, Department of Thermal Engineering, Tsinghua University, Beijing, China \\ ayangyz14@mails.tsinghua.edu.cn, bjiangdx@mail.tsinghua.edu.cn
}

Keywords: numerical integration; trend term; fault diagnosis; empirical mode decomposition (EMD)

\begin{abstract}
Acceleration signal is widely used in fault diagnosis of power machine, which can be numerical integrated to obtain displacement data. DC components and trend terms can't be avoided in acceleration signal and it will be magnified during integration, causing distortion of the displacement waveform acquired. To eliminate the influence of the trend term, the method of high-pass frequency domain filtering, polynomial curve fitting and empirical mode decomposition (EMD) are tried in this paper to deal with the experimental data collected from our rotor-bearing-casing coupled vibration experiment system. EMD shows its significant effect extracting the trend of displacement data integrated from the acceleration.
\end{abstract}

\section{Introduction}

To process and analyze signals collected from vibration sensors is one of the most common way of power machine's fault diagnosis [1]. In many situations we cannot set up displacement sensors inside a machine and gain displacement signal directly because of its complex structure or other limits. Accelerometers can easily set up outside casings, bearing supports and gear boxes. It is a practical way to obtain displacement history curves by double integration of acceleration signals. Affected by gravity or the instability of sensors, DC components and trend terms can't be avoided in acceleration signals. They must be eliminated out of the signals otherwise after integration the error will be accumulated and the displacement wave we get will be totally distorted.

There are several ways often used in eliminating trend terms of signals including average slope method, difference method, polynomial fitting, high-pass filtering [2]. Trend terms with certain regularity like linear trend, polynomial trend and exponential trend can be removed with relative accuracy. These types of trend assumed in the methods may not be the real situation. When dealing with experimental or practical data, more advanced method should applied.

Empirical mode decomposition (EMD) can decompose a signal without the assumption of any trend term function, suitable to analyze non-linear and non-stationary signals and has remarkable effect dealing with signal's trend term [1]. Recently the EMD method is widely applied in the area of signal analysis and fault diagnosis [3,4]. In this paper, several methods including empirical mode decomposition are used to eliminate the complex trend term off the experimental data collected from the rotor-bearing-casing coupled vibration experiment system, during the process of numerical integration to get displacement data from the acceleration signal, and the results are compared.

\section{Integration in time domain and in frequency domain}

There are two ways of numerical integration to transform acceleration signal into displacement signal. One is to integrate the acceleration directly in time domain for two times, which first yields the velocity then the displacement. The other is to get the Fourier coefficient of the displacement from the Fourier-transformed acceleration signal, then take the inverse Fourier transform [5].

The time domain component of the acceleration at any frequency $\omega$ can be expressed as the following Eq.1 according to the formula of inverse Fourier transform.

$$
a(t)=A e^{j \omega t} \text {. }
$$


The frequency component of velocity and displacement can be described as Eq.2 and Eq.3 [6].

$$
\begin{aligned}
& v(t)=\int_{0}^{t} a(\tau) d \tau=\int_{0}^{t} A e^{j \omega t} d \tau=\frac{A}{j \omega} e^{j \omega t}=V e^{j \omega t}, \\
& x(t)=\int_{0}^{t}\left[\int_{0}^{t} a(\tau) d \tau\right] d \lambda=\int_{0}^{t} V e^{j \omega t} d \lambda=\frac{V}{j \omega} e^{j \omega t}=\frac{A}{-\omega^{2}} e^{j \omega t}=X e^{j \omega t} .
\end{aligned}
$$

So the Fourier coefficient of the displacement will be given by dividing the Fourier-transformed acceleration signal by $-\omega^{2}$.

The method of integration in time domain is taken used of in this paper since the results of the two method appear little difference dealing the sample signal and the way trend accumulating will be watched during the time domain integration. The result of integration in frequency domain can be a reference.

\section{Experimental Device and sample signal}

The rotor-bearing-casing coupled vibration experiment system is designed in our laboratory to simulate different fan casing vibration faults of aero-engine. Because of the strict demand of aero-engine's structure, displacement sensors can't installed inside the casing to monitor the vibration situation of the shaft. So accelerometers will be set up outside the casing to collect the vibration acceleration signal. To study the transmission characteristics of the mechanical vibration from the shaft to the casing, accelerometers are also set up outside the casing of our experimental device.
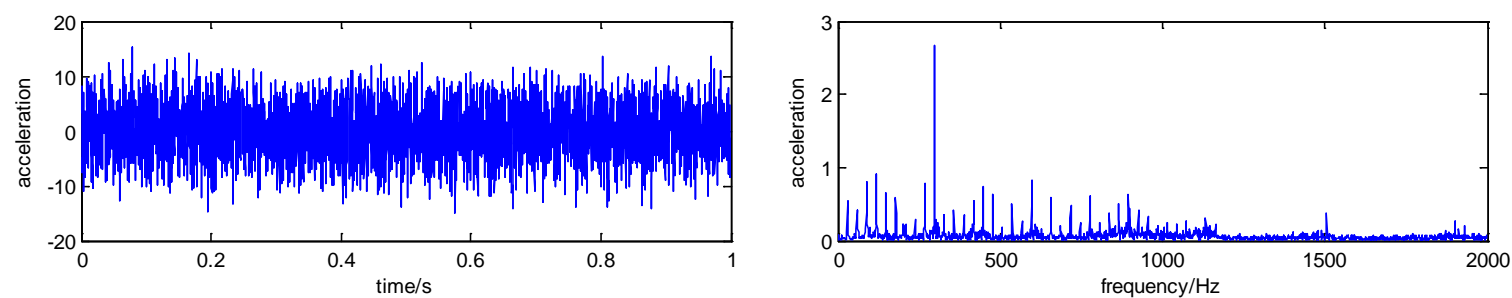

Fig. 1 The sample signal and its frequency spectrum.
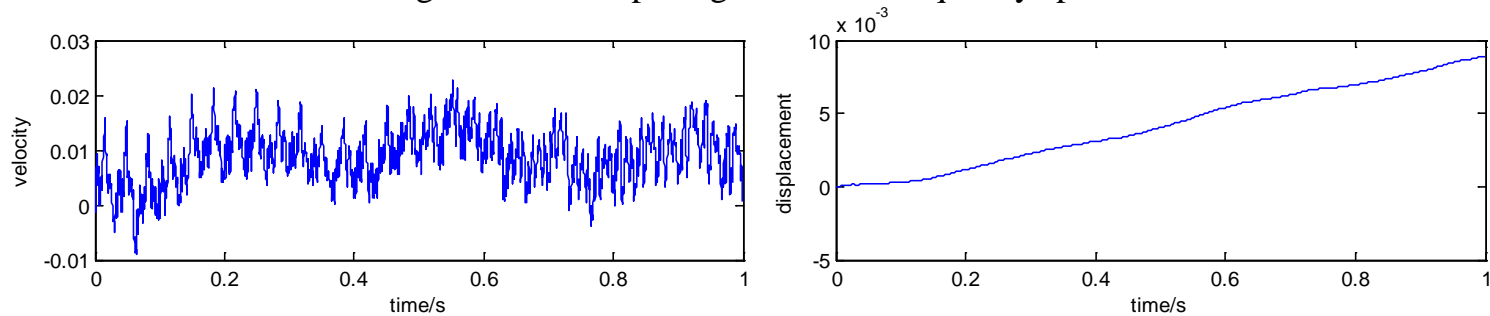

Fig. 2 The velocity and displacement waveform integrated from the acceleration signal.

The sample signal shown in Fig.1 was collected from one of the accelerometers on the fan casing at the sampling frequency of $8000 \mathrm{~Hz}$ for $1 \mathrm{~s}$. Some level of imbalance fault was added and the rotational speed of the shaft was set as about $1800 \mathrm{rpm}(30 \mathrm{~Hz})$. Before any analysis the mean value had been subtracted from the acceleration signal, aiming to get rid of the effect of DC component. The velocity data is obtained by integrating the acceleration signal in time domain for one time and the displacement data for two times (Fig.2). Obvious trend term can't be seen from the acceleration signal. But after one time integration the trend is accumulated, causing the big fluctuation of the velocity data. The displacement waveform acquired from double integration is largely distorted by the trend and the information is flooded by the accumulated error.

\section{High-pass frequency domain filtering method}

The trend term can be seen as the low frequency part of the signal including the $0 \mathrm{~Hz}$ component. If the sampling frequency are not the integer multiples of the main period of the signal, the mean 
value may not equals to the $\mathrm{OHz}$ part. So subtracting the mean value may can't eliminating the DC component effectively. Filtering the low frequency part as well as the $0 \mathrm{~Hz}$ component in frequency domain can be a valid method to eliminate the trend term.

To achieve the effect of ideal High-pass frequency domain filtering, we can take the fast Fourier transform (FFT) of the signal to get the frequency data, set the low frequency part as 0 , and do inverse fast Fourier transform to turn the signal back into time domain. In this way the low frequency component which was set to 0 will not accumulate during the integration and then the trend term eliminated. Fig. 3 shows the displacement time histories integrated from the acceleration signal of which the low frequency part no more than $0 \mathrm{~Hz}, 1 \mathrm{~Hz}, 3 \mathrm{~Hz}$ and $6 \mathrm{~Hz}$ were subtracted. We can see that more low frequency part subtracted, more trend term eliminated, and closer the waveform to the traditional displacement vibration signal.
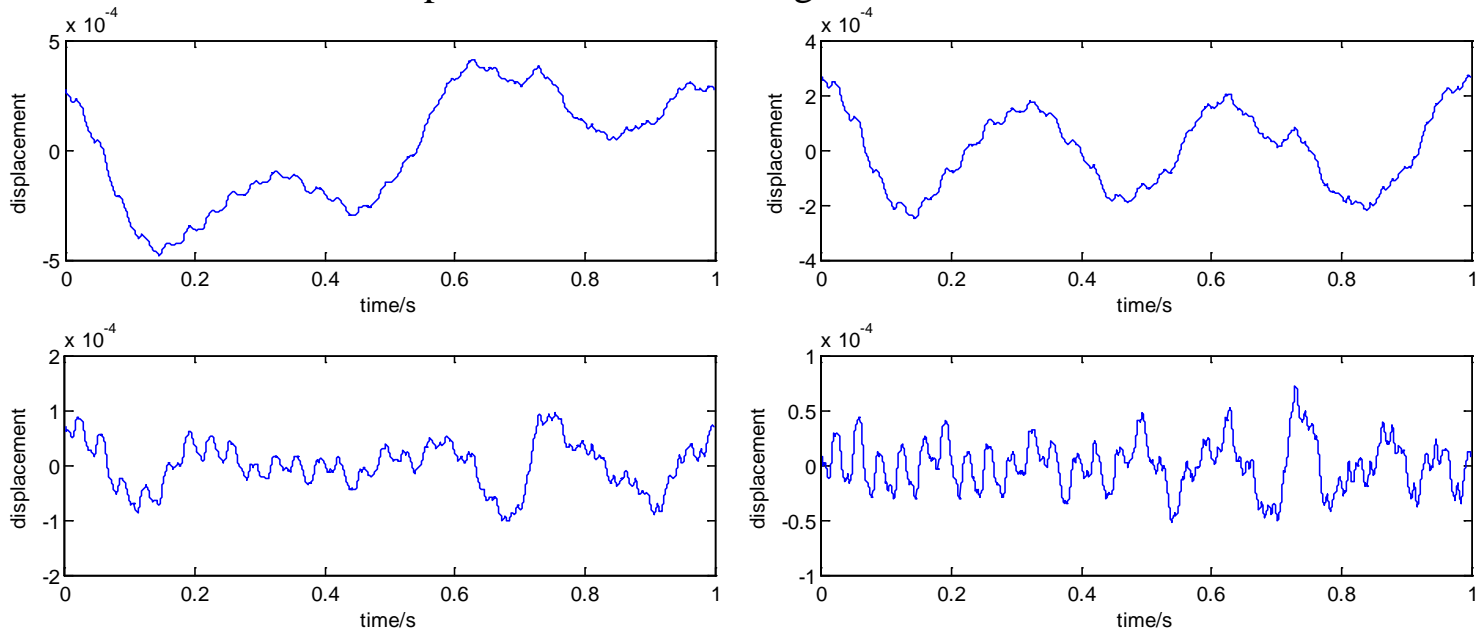

Fig. 3 The displacement time histories with no more than $0 \mathrm{~Hz}, 1 \mathrm{~Hz}, 3 \mathrm{~Hz}$ and $6 \mathrm{~Hz}$ subtracted.

However, using this method the information of the low frequency part subtracted will completely lost. And the amount of frequency component to set 0 is hard to decide because the larger the amount is, the more trend term wiped off but the more information lost. Form the frequency spectrum in Fig.4 we can see that too much information will be lost if we want to eliminate the trend term completely using this method (almost delete all the frequency component below the rotational speed).
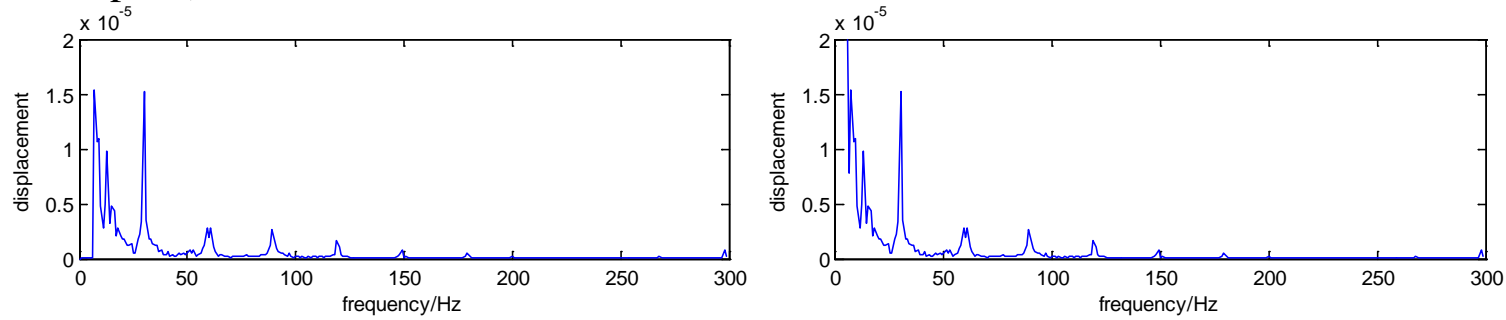

Fig. 4 Displacement spectrum with $6 \mathrm{~Hz}$ subtracted (left) and the one of frequency domain integration (right).

\section{Polynomial curve fitting method}

Polynomial curve fitting method uses the least square method to acquire polynomial fitted curve of the data [7]. The curve is thought to near the mean value line of the signal which can be treated as the trend term. If the trend term of the signal is close to DC component, straight line, quadratic curve or any Polynomial curve, it can be removed easily with this method. Fig.5 shows the 9-order polynomial fitting curve of the acceleration signal. 


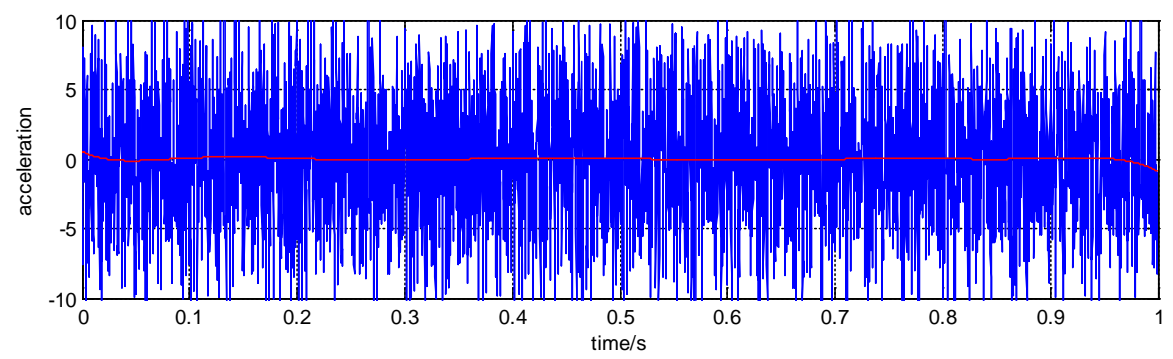

Fig. 5 The 9-order polynomial fitting curve of the acceleration signal.

Different orders of polynomial curves are tried fitting the acceleration waveform. Fig. 6 shows the result after 9-order and 15-order polynomial fitting. The mean value which doesn't equal to 0 results in the drift of the displacement. The result of which the $0 \mathrm{~Hz}$ component is removed after each integration is shown in Fig.7. This time the result seems good but some low frequency fluctuation still can't be removed clear.
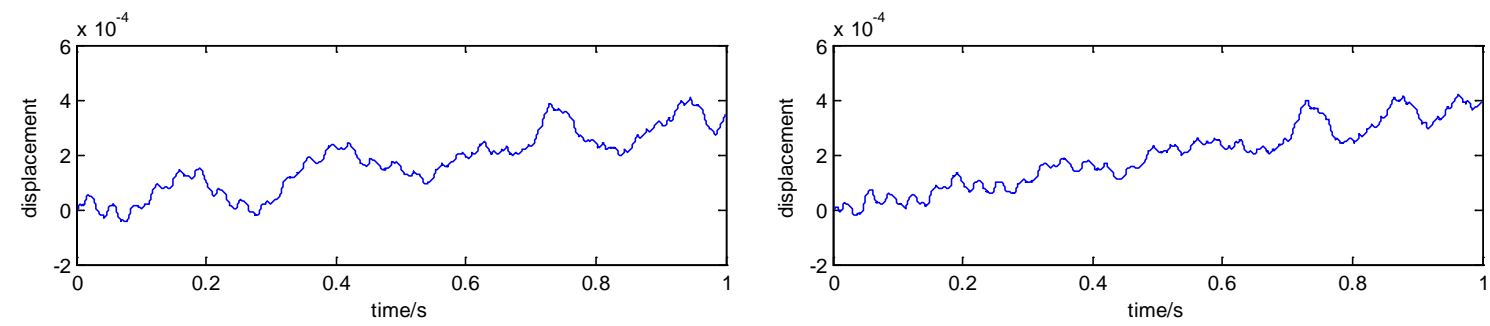

Fig. 6 The displacement after 9-order and 15-order polynomial fitting.
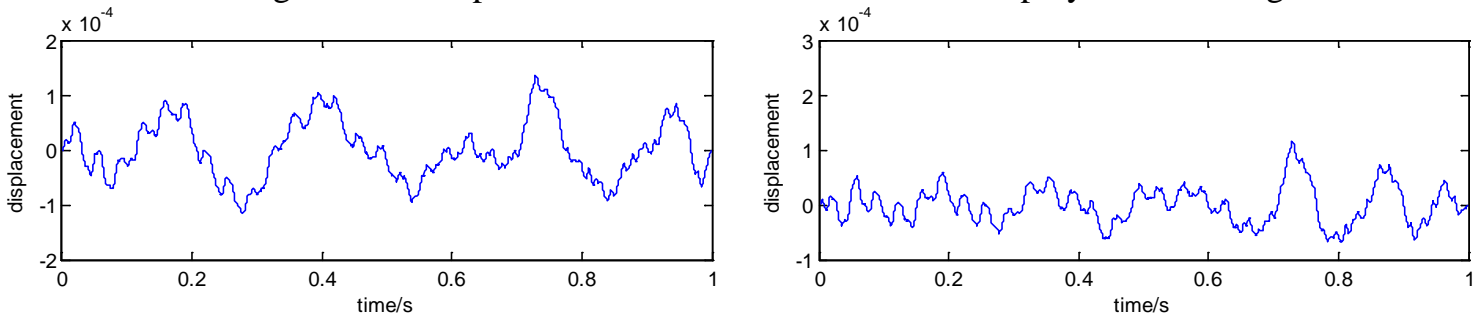

Fig. 7 The displacement after 9-order and 15-order polynomial fitting ( $0 \mathrm{~Hz}$ removed).

\section{Empirical mode decomposition method}

Empirical mode decomposition (EMD) is a very useful method developed for the analysis of non-stationary signals in time-frequency domain. It's based on the assumption that any signal can be composed by several zero-mean AM-FM components which is called intrinsic mode functions (IMFs). It can be expressed as the following equation where $x(t)$ stands for the signal, $\operatorname{im} f_{i}(t)$ for the IMFs and $r_{n}(t)$ for the residue.

$$
x(t)=\sum_{i=1}^{n} i m f_{i}(t)+r_{n}(t),
$$

The principle of EMD can be seen in details in [8]. In this paper we take advantage of the MATLAB program written by Gabriel Rilling and his team [9].

The residue $r_{n}(t)$ is thought to be the DC component or trend term of the signal. Doing EMD to the acceleration signal, distracting $r_{n}(t)$ from $x(t)$ and taking double integrations, the result with $0 \mathrm{~Hz}$ component removed after each integration is shown in Fig.8. This method doesn't work well since the low frequency trend not removed is amplified after integrations, which means the trend term doesn't remain in the residue only but also distributed in the IMFs. Even subtracting last few low frequency IMFs from the acceleration signal doesn't make the result better. 


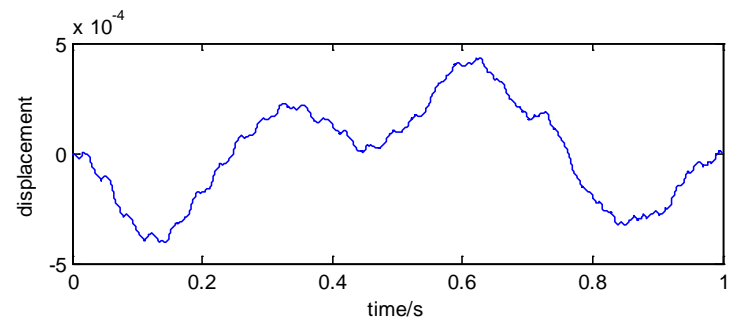

Fig. 8 The displacement with EMD residue subtracted before integration and $0 \mathrm{~Hz}$ removed.

Then EMD is taken to the displacement data acquired by double integration with no other treatment (Fig.9). The displacement waveform with the residue subtracted comes out a remarkable result of trend term elimination. The final displacement time history appears the close look to a common wave and its spectrum shows the main frequency component clearly (Fig.10), which is very close to the spectrum of displacement integrated in frequency domain (Fig.4 right).
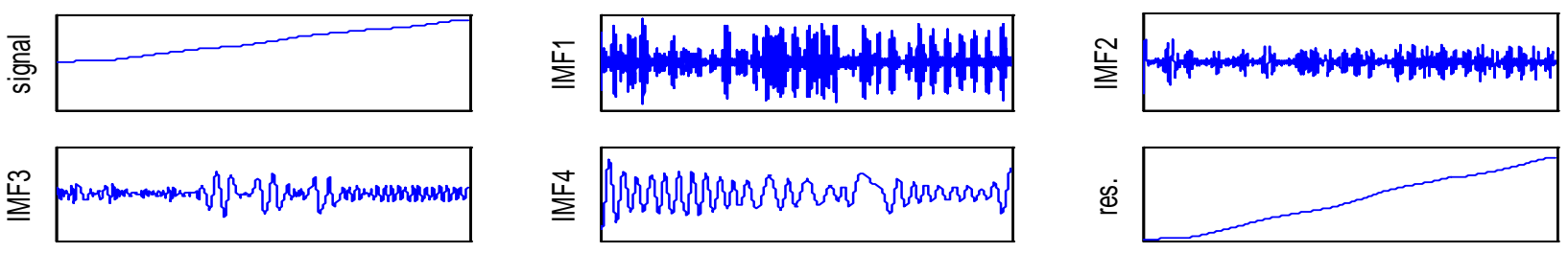

Fig. 9 The result of EMD of the displacement with no other treatment.
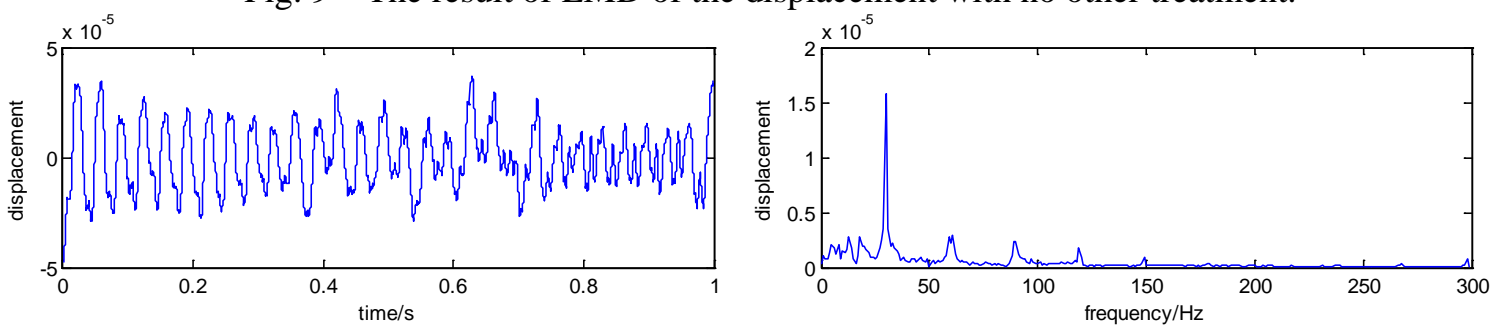

Fig. 10 The displacement with EMD residue subtracted after integration and its frequency spectrum.

Here in Fig.11 and Fig.12 are two more examples of different vibration situation treated by this method. Some degree of misalignment or looseness fault were added. The trend terms are also well eliminated. In some cases when the signal fluctuates greatly, not just the residue of EMD, but the last one or two low frequency IMFs should be subtracted in order to remove the trend term clear. Then some of the low frequency information may be lost, and that makes the EMD method not a perfect way of trend term elimination, which means other methods not mentioned in this paper may be needed handling this kind of situation.
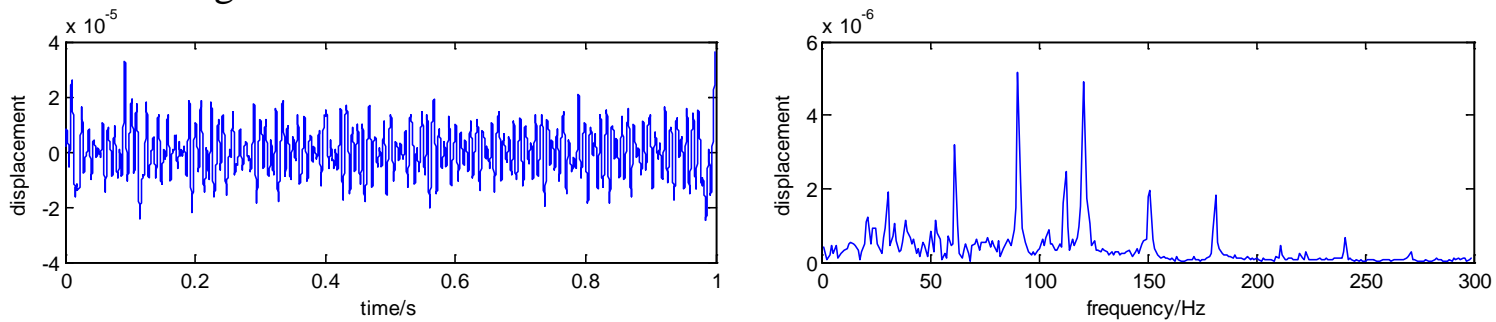

Fig. 11 Result of the example with some misalignment fault.
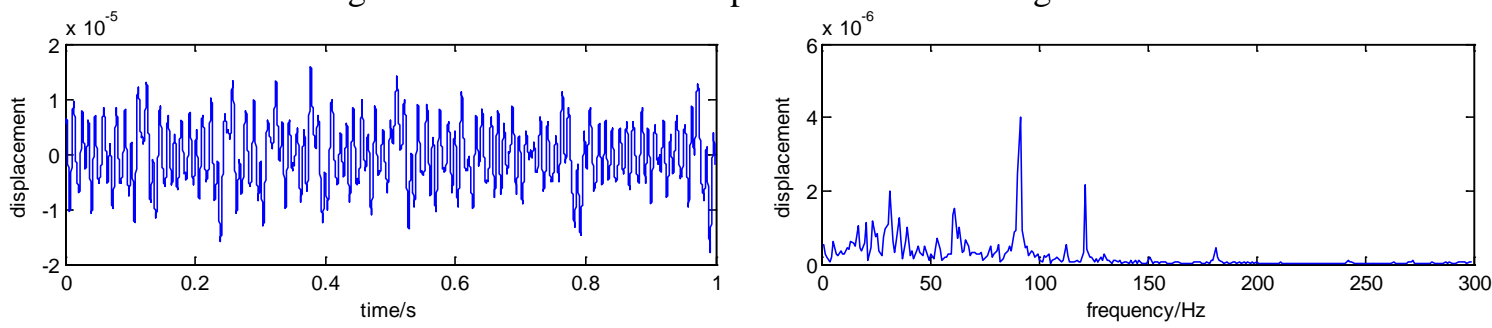

Fig. 12 Result of the example with some looseness fault.

\section{Conclusion}

To eliminate the complex trend term off the experimental data collected from the vibration fault 
diagnosis experimental device, several methods are used including high-pass frequency domain filtering, polynomial curve fitting and empirical mode decomposition. High-pass frequency domain filtering method does well in wiping off the low frequency fluctuation but will cause the loss of some information. Polynomial curve fitting method will yield good result when the trend is linear or polynomial, but gain unsatisfied effect as the trend term is complex. Taking EMD before double integration cannot eliminate the trend term clear. But when the acceleration signal is integrated twice and the trend is magnified, clear waveform of displacement is acquired taking EMD and subtracting the residue.

\section{References}

[1] He Zheng-jia, Chen Jin, Wang Tai-yong, Chu Fu-lei. Theories and Applications of Machinery Fault Diagnosis [M]. Higher Education Press, 2010. (in Chinese)

[2] Chen Jun, Xu You-lin. Application of EMD to Signal Trend Extraction [J]. Journal of Vibratio n, Measurement\& Diagnosis, 2005, 25(2):101-104. DOI:10.3969/j.issn.1004-6801.2005.02.005. (in Chinese)

[3] Ali J B, Fnaiech N, Saidi L, et al. Application of empirical mode decomposition and artificial neural network for automatic bearing fault diagnosis based on vibration signals [J]. Applied Acoustics, 2015, 89:16-27.

[4] Liu B, Riemenschneider S, Xu Y. Gearbox fault diagnosis using empirical mode decomposition and Hilbert spectrum [J]. Mechanical Systemss\&ssignal Processing, 2006, 20(3):718 - 734.

[5] Han S. Measuring displacement signal with an accelerometer [J]. Journal of Mechanical Science \& Technology, 2010, 24(6):1329-1335.

[6] FANG Xin-lei, HAO Wei, CHEN Hong. Acceleration Signal Processing Based on Frequency Domain Filtering [J]. Instrument Technique and Sensor, 2012, (4):94-96. DOI:10.3969/j.issn.1002-1841.2012.04.034. (in Chinese)

[7] LI Dong-wen, XIONG Xiao-yan, LI Bo. Research on the Processing of Vibration Acceleration Signal [J]. Mechanical \& Electrical Engineering Technology, 2008, 37(9):50-52. DOI:10.3969/j.issn.1009-9492.2008.09.019. (in Chinese)

[8] Huang N E, Shen Z, Long S R, et al. The empirical mode decomposition and the hilbert spectrum for non-linear and non-stationary time series analysis [J]. Royal Society of London Proceedings, 1998, 454(1971):903.

[9] Rilling G, Flandrin P, Gonçalvès P. On Empirical Mode Decomposition and its Algorithms [C]. //Proc IEEE Eurasip Workshop on Nonlinear Signal \& Image Processing Nsip Grado. 2003. 